\title{
Low-mass star formation in R Coronae Australis: observations of organic molecules with the APEX telescope
}

\author{
F. L. Schöier ${ }^{1}$, J. K. Jørgensen ${ }^{2}$, K. M. Pontoppidan ${ }^{3}$, and A. A. Lundgren ${ }^{4}$ \\ 1 Stockholm Observatory, AlbaNova University Center, 10691 Stockholm, Sweden \\ e-mail: fredrik@astro.su.se \\ 2 Harvard-Smithsonian Center for Astrophysics, 60 Garden Street MS42, Cambridge, MA 02138, USA \\ 3 Division of Geological and Planetary Sciences, California Institute of Technology, MS 150-21, Pasadena, CA 91125, USA \\ ${ }^{4}$ European Southern Observatory, Casilla 19001, Santiago 19, Chile
}

Received 31 March 2006 / Accepted 15 May 2006

ABSTRACT

\begin{abstract}
This paper presents new APEX submillimetre molecular line observations of three low-mass protostars, IRS7A, IRS7B, and IRAS32, in the R Coronae Australis molecular cloud complex. The molecular excitation analysis is performed using a statistical equilibrium radiative transfer code. The derived beam averaged fractional abundances vary by less than a factor of two among the three sources, except those of $\mathrm{H}_{2} \mathrm{CO}$ and $\mathrm{CH}_{3} \mathrm{OH}$, which show differences of about an order of magnitude. The molecular abundances are similar to those typically found in other star-forming regions in the Galaxy, such as the $\rho$ Oph and Perseus molecular clouds. There is a marked difference in the kinetic temperatures derived for the protobinary source IRS7 from $\mathrm{H}_{2} \mathrm{CO}(\approx 40-60 \mathrm{~K})$ and $\mathrm{CH}_{3} \mathrm{OH}(\approx 20 \mathrm{~K})$, possibly indicating a difference in origin of the emission from these two molecules.
\end{abstract}

Key words. stars: formation - ISM: molecules - ISM: abundances - astrochemistry

\section{Introduction}

In the earliest, deeply-embedded stage, low-mass protostars are surrounded by a collapsing envelope and a circumstellar disk. In this phase, the initial chemical composition of the disk is determined. The chemistry of molecules in the circumstellar envelope is likely to be directly reflected in the molecular composition in the disk, which forms the building blocks from which planets may form. An interesting aspect is the search for low-mass "hot cores", i.e., regions where grain ice mantles are liberated in the envelope close to the central protostar because of its heating (e.g., Ceccarelli et al. 2000; Schöier et al. 2002; Cazaux et al. 2003). The search for hot cores around low-mass stars is not yet conclusive, mainly due to limitations in the available observational data. Also, outflows are likely to play an important role by affecting many of the same species thought to be sign posts of hot cores (e.g., Jørgensen et al. 2005). Moreover, the chemistry of traditional hot-core tracers such as $\mathrm{H}_{2} \mathrm{CO}$ may be affected by the freeze-out of CO (e.g., Schöier et al. 2004), which is why combined studies of chemically related molecular species are important.

The R Coronae Australis dark cloud is a nearby (170 pc, Knude \& Høg 1998) region of active star formation (e.g., Brown 1987; Chini et al. 2003; Nutter et al. 2005). Molecular line observations of protostellar objects in R Coronae Australis have attained little attention, mainly due to its location on the southern hemisphere. Previous studies using single-dish telescopes with limited spatial resolution, which are mainly sensitive to the chemistry of the outer cold regions of the envelopes, have been biased towards lower excitation lines. A wealth of molecular rotational transitions lying in the $279-381 \mathrm{GHz}$ window are accessible with APEX ${ }^{1}$, and can be used to constrain the physics, and in particular, the chemistry in the dense $\left(>10^{6} \mathrm{~cm}^{3}\right)$ and warm $(>30 \mathrm{~K})$ material in the envelopes. Observations of protostellar objects in Corona Australis are also interesting for testing the importance of the surroundings on the formation of protostars, and will complement previous extensive studies in Perseus and $\rho$ Oph (Jørgensen et al. 2002, 2004, 2005). It is not clear how the properties of the parental cloud affect (are reflected in) the properties of the emerging protostar (see Jayawardhana et al. 2001). Likewise, the properties of the parental cloud may reflect the chemical evolution of the protostar, for example, in the degree of freeze-out and the "initial abundances".

The first step in a project aimed at obtaining an extensive data base of molecular line emission towards a large sample of protostars in the southern hemisphere, with a focus on the Corona Australis and Chameleon molecular clouds, is presented in this letter. It is crucial to utilize APEX to build comparable samples of well-studied low-mass protostars for future ALMA observations.

\section{Observations}

Observations of sub-millimetre molecular line emission towards the R Coronae Australis molecular cloud complex were performed September 4-6, 2005, using the $12 \mathrm{~m}$ APEX telescope equipped with an SIS receiver (APEX-2a). Typical system temperatures were in the range $140-380 \mathrm{~K}$ (DSB). The $1 \mathrm{GHz}$ FFTS

1 This publication is based on data acquired with the Atacama Pathfinder Experiment (APEX). APEX is a collaboration between the Max-Planck-Institut für Radioastronomie, the European Southern Observatory, and the Onsala Space Observatory. 

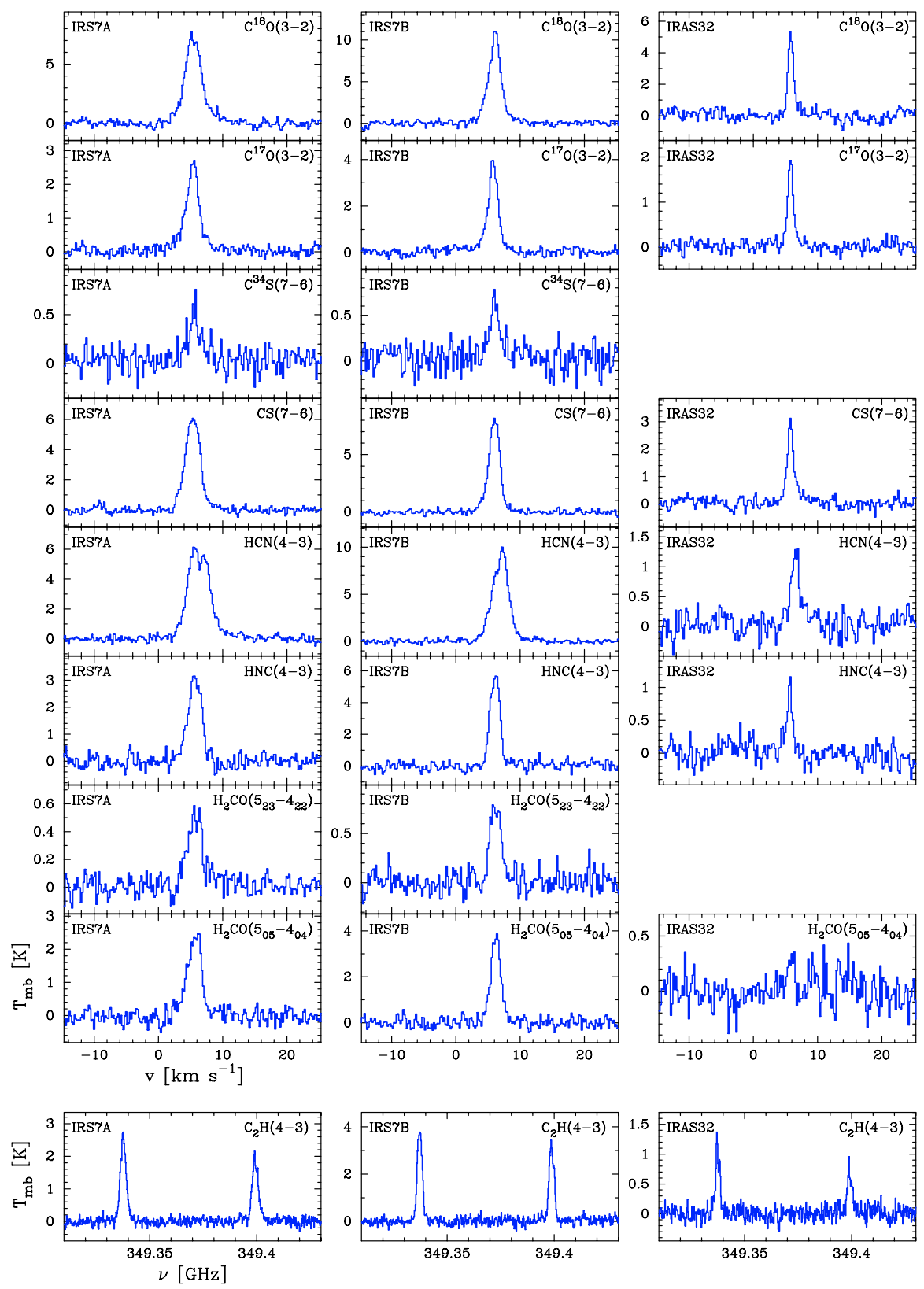

Fig. 1. New observations of molecular line emission using the APEX telescope. The velocity resolution is $0.2 \mathrm{~km} \mathrm{~s}^{-1}$ in all the spectra.

with 8192 spectral channels was used as a backend, providing a spectral resolution of $122 \mathrm{kHz}\left(0.1 \mathrm{~km} \mathrm{~s}^{-1}\right)$. The observations were carried out using a position-switching mode, with the reference position located at $+2^{\prime}$ in azimuth. The data were reduced in a standard way by removing, in most cases, a low order $(\leq 3)$ polynomial baseline and the raw spectra, stored in the $T_{\mathrm{A}}^{*}$ scale, converted to main-beam brightness temperature using $T_{\mathrm{mb}}=T_{\mathrm{A}}^{*} / \eta_{\mathrm{mb}} . T_{\mathrm{A}}^{*}$ is the antenna temperature corrected for atmospheric attenuation using the chopper-wheel method, and $\eta_{\mathrm{mb}}$ is the main-beam efficiency. A value of $\eta_{\mathrm{mb}}=0.7$ was assumed for all spectral settings. Regular pointing checks were made on strong CO sources and typically found to be consistent within $\approx 3^{\prime \prime}$. The FWHM of the main beam is $18^{\prime \prime}$ at $345 \mathrm{GHz}$. The uncertainty (including errors in pointing, calibration, and $\eta_{\mathrm{mb}}$ ) in the absolute intensity scale (main-beam brightness) is estimated to be about $\pm 15 \%$.
The observed spectra for the protostellar objects IRS7A $\left(\alpha_{2000}=19: 01: 55.3, \delta_{2000}=-36: 57: 21\right)$, IRS7B $\left(\alpha_{2000}=\right.$ $\left.19: 01: 56.4, \delta_{2000}=-36: 57: 27\right)$, and IRAS32 $\left(\alpha_{2000}=\right.$ 19:02:58.7, $\left.\delta_{2000}=-37: 07: 34\right)$ are presented in Figs. 1 and 2 (the positions are obtained from IRAC4 $8 \mu \mathrm{m}$ pipeline data in the Spitzer Space Telescope archive: AOR ID 3650816). IRS7B is identified as a class 0 protostar, whereas IRS7A and IRAS32 are of class I (see Nutter et al. 2005, and references therein).

Results of Gaussian fits to the spectra are reported in Table 1. The systemic (LSR) velocities obtained from the Gaussian fits are 5.3, 5.6, and $5.9 \mathrm{~km} \mathrm{~s}^{-1}$ (LSR) for IRS7A, IRS7B, and IRAS32, respectively. The spectra are generally well represented by a single Gaussian, with the major exception being the observed HCN $J=4 \rightarrow 3$ line emission towards IRS7A and IRS7B. In these two cases, not only is the HCN line significantly broader, compared to other molecular lines, but also a central 


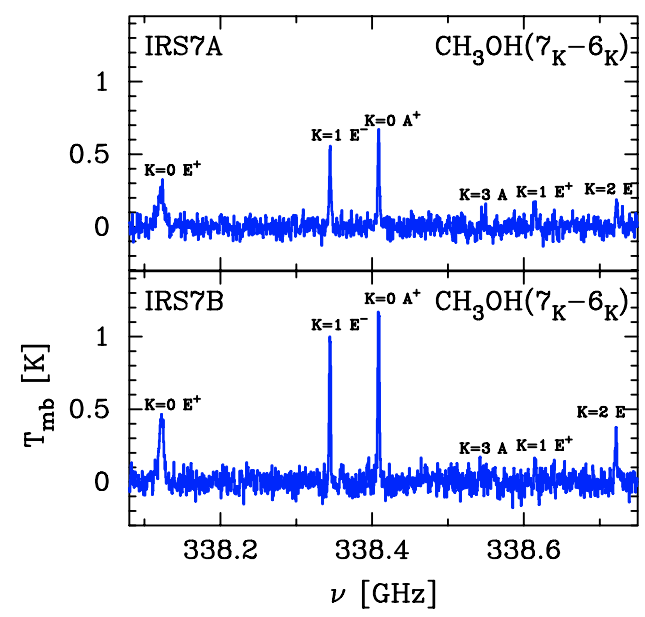

Fig. 2. New observations of $\mathrm{CH}_{3} \mathrm{OH}$ line emission using the APEX telescope. The velocity resolution is $0.5 \mathrm{~km} \mathrm{~s}^{-1}$ in both spectra. Note that the $7_{0} \rightarrow 6_{0} \mathrm{E}^{+}$line is blended with the $\mathrm{CH}_{3} \mathrm{OH} 4_{0} \rightarrow 3_{1} \mathrm{E}^{-}$line from the upper sideband.

Table 1. Observational results ${ }^{a}$.

\begin{tabular}{|c|c|c|c|c|c|c|c|c|c|}
\hline \multirow[b]{2}{*}{ Transition } & \multicolumn{3}{|c|}{ IRS7A } & \multicolumn{3}{|c|}{ IRS7B } & \multicolumn{3}{|c|}{ IRAS 32} \\
\hline & $I$ & $T_{\mathrm{mb}}$ & $\Delta v$ & $I$ & $T_{\mathrm{mb}}$ & $\Delta v$ & $I$ & $T_{\mathrm{mb}}$ & $\Delta v$ \\
\hline $\mathrm{C}^{18} \mathrm{O}$ & 24.9 & 7.2: & 3.1: & 26.9 & 10.2: & 2.3: & 6.5 & 5.2 & 1.0 \\
\hline $\mathrm{C}^{17} \mathrm{O}_{3}$ & 7.5 & 2.5: & 2.5: & 8.8 & 3.7: & 1.9: & 2.6 & 1.9 & 1.0 \\
\hline$C^{34} S$ 7-6 & 1.4 & 0.47 & 2.5 & 2.0 & 0.61 & 2.1 & $<0.2$ & $\ldots$ & $\ldots$ \\
\hline CS 7-6 & 17.6 & 6.2 & 2.7 & 17.6 & 8.1 & 2.0 & 4.6 & 2.8: & 1.3: \\
\hline $\mathrm{HCN}$ & 26.0 & 6.1: & 3.9: & 30.4 & 9.2: & 3.0: & 2.7 & 1.2: & 1.8: \\
\hline HNC & 8.1 & 3.1 & 2.4 & 11.3 & 5.9 & 1.8 & 1.8 & 1.1: & 1.0: \\
\hline \multicolumn{10}{|l|}{$\mathrm{C}_{2} \mathrm{H}:$} \\
\hline $4_{5}-3_{4}$ & 7.7 & 2.6 & 2.8 & 10.0 & 4.0 & 2.3 & 2.4 & 1.1: & 2.1: \\
\hline$-3_{3}$ & 6.4 & 1.9 & 2.9 & 8.4 & 2.2 & 2.4 & 2.1 & 0.7: & 2.4: \\
\hline \multicolumn{10}{|l|}{$\mathrm{H}_{2} \mathrm{CO}:$} \\
\hline $5_{23}-4_{22}$ & 1.8 & 0.51 & 2.8 & 1.7 & 0.81 & 2.0 & $<0.2$ & 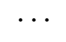 & 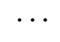 \\
\hline $5_{05}-4_{04}$ & 6.2 & 2.4 & 2.5 & 7.7 & 3.8 & 1.8 & 0.3: & $\ldots$ & . \\
\hline \multicolumn{10}{|l|}{$\mathrm{CH}_{3} \mathrm{OH}:$} \\
\hline $7_{0}-6_{0} \mathrm{E}^{+b}$ & 2.3 & $\cdots$ & $\cdots$ & 3.0 & $\cdots$ & $\cdots$ & $<0.1$ & $\cdots$ & \\
\hline $6 . \mathrm{F}^{-}$ & 1.5 & 0.50 & 2.6 & 2.2 & 1.0 & 2.1 & $<0.1$ & $\ldots$ & \\
\hline $7_{1}-6_{1} \mathrm{E}^{\mathrm{T}}$ & 0.49 & $\ldots$ & $\cdots$ & 0.37 & $\ldots$ & $\ldots$ & $<0.1$ & $\ldots$ & \\
\hline $7_{2}-6_{2} \mathrm{E}^{ \pm}$ & 0.51 & $0.18:$ & 3.0: & 0.90 & 0.35: & 2.6: & $<0.1$ & $\ldots$ & 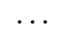 \\
\hline $7_{0}-6_{0}$ & 1.8 & 0.64 & 2.4 & 3.3 & 1.2 & 2.3 & $<0.1$ & $\cdots$ & .. \\
\hline $7_{3}-6_{3} \mathrm{~A}^{ \pm}$ & 0.2 : & $\ldots$ & $\ldots$ & 0.2 : & $\ldots$ & $\ldots$ & $<0.1$ & $\ldots$ & \\
\hline
\end{tabular}

${ }^{a} I=\int T_{\mathrm{mb}} \mathrm{d} v$ is the integrated intensity in $\mathrm{K} \mathrm{km} \mathrm{s}^{-1} \cdot T_{\mathrm{mb}}$ is the peak intensity (in $\mathrm{K}$ ) and $\Delta v$ the $F W H M$ (in $\mathrm{km} \mathrm{s}^{-1}$ ) from Gaussian fits. A colon (:) marks an uncertain value due to low a $\mathrm{S} / \mathrm{N}$-ratio or a significant departure from a Gaussian line profile. ${ }^{b}$ Blended with the $\mathrm{CH}_{3} \mathrm{OH} 4_{0} \rightarrow 3_{1} \mathrm{E}^{-}$line from the upper sideband. The integrated intensity refers to the sum of both lines.

absorption dip is present, at least in the IRS7A spectrum. The $\mathrm{HCN}$ spectra presented here are very similar to those of $\mathrm{HCO}^{+}$ $J=4 \rightarrow 3$ obtained by Anderson et al. (1997a). They interpret the emission as arising in a large rotating (circumbinary) disk, and the self-absorption feature is caused by absorption in a dense and quiescent foreground of the cloud. In addition, for the two IRS7 sources, our observed $\mathrm{C}^{17} \mathrm{O}$ and, in particular, $\mathrm{C}^{18} \mathrm{O}$, $J=3 \rightarrow 2$ spectra have weak wings, possibly related to the known molecular outflow in this region, and are somewhat better reproduced by a sum of two Gaussians.

\section{Excitation analysis}

Presently, there is a lack of observational data required to carry out a detailed modelling of the dust emission, mainly due to limited spatial resolution (see Nutter et al. 2005). This is usually the preferred method to determine the physical structure, such as density and temperature, of material present around protostars that is required for a detailed analysis of its molecular line emission (e.g., Jørgensen et al. 2002).

A first estimate of the excitation conditions can be obtained assuming the lines to be emanating from the same homogeneous region. We have adopted RADEX, a statistical equilibrium radiative transfer code that uses an escape probability formalism to calculate the excitation and emission from the protostellar cores in our sample. RADEX is comparable to the LVG method and provides a useful tool for rapidly analysing a large set of observational data providing constraints on physical conditions, such as density and kinetic temperature (Jansen et al. 1994; van der Tak et al., in prep.). An online version of RADEX, together with relevant molecular data used in the excitation analysis and summarized in Schöier et al. (2005), is made publicly available through the Leiden Atomic and Molecular Database $(\text { LAMDA })^{2}$.

In the excitation analysis, the number density of $\mathrm{H}_{2}$ molecules was fixed to $3 \times 10^{6} \mathrm{~cm}^{-3}$, typical of the warmer, more dense regions of the condensed cores. From the two lines of $\mathrm{H}_{2} \mathrm{CO}$, the kinetic temperatures were estimated to be $\approx 60 \mathrm{~K}$ and $\approx 40 \mathrm{~K}$ for IRS7A and IRS7B, respectively. The corresponding column densities derived were $2 \times 10^{13} \mathrm{~cm}^{-2}$ (IRS7A) and $3.5 \times 10^{13} \mathrm{~cm}^{-2}$ (IRS7B). In the case of $\mathrm{CH}_{3} \mathrm{OH}$, a full analysis using a $\chi^{2}$-statistic was used, and the kinetic temperatures derived were $23 \pm 3 \mathrm{~K}$ and $22 \pm 3 \mathrm{~K}$ for IRS7A and IRS7B, respectively. The column densities of $\mathrm{CH}_{3} \mathrm{OH}$ derived were $1.4 \pm 0.4 \times 10^{14} \mathrm{~cm}^{-2}$ (IRS7A) and $1.8 \pm 0.4 \times 10^{14} \mathrm{~cm}^{-2}$ (IRS7B). The substantial difference in the derived kinetic temperatures from $\mathrm{H}_{2} \mathrm{CO}$ and $\mathrm{CH}_{3} \mathrm{OH}$ could possibly indicate different origins for these traditional hot-core tracers.

To determine the column densities for the remaining molecular species, we adopted a kinetic temperature of $40 \mathrm{~K}$. The column densities obtained for $\mathrm{C}^{34} \mathrm{~S}$ in the case of IRS7A $(1 \times$ $\left.10^{13} \mathrm{~cm}^{-2}\right)$ and IRS7B $\left(1.5 \times 10^{13} \mathrm{~cm}^{-2}\right)$ were used together with a "standard" fractional abundance $\left(5 \times 10^{-11}\right)$ of $C^{34} \mathrm{~S}$ (Jørgensen et al. 2004, see Table 2) to estimate the total $\mathrm{H}_{2}$ column densities. In the case of IRS7A, we obtain a $\mathrm{H}_{2}$ column density of $2 \times 10^{23} \mathrm{~cm}^{-2}$, and for IRS7B, a slightly higher value of $3 \times 10^{23} \mathrm{~cm}^{-2}$. For IRAS32, no $\mathrm{C}^{34} \mathrm{~S} J 7 \rightarrow 6$ line emission was detected, and instead the main isotope (with an adopted fractional abundance of $1 \times 10^{-9}$ ) was used to estimate the $\mathrm{H}_{2}$ column density to $3 \times 10^{22} \mathrm{~cm}^{-2}$. The derived fractional abundances for all molecular species observed are reported in Table 2 .

\section{Results and discussion}

The excitation analysis gives results (see Table 2) for the abundances of common molecules such as $\mathrm{CO}, \mathrm{CS}$, and $\mathrm{HNC}$, and for more complex species, such as $\mathrm{H}_{2} \mathrm{CO}$ and $\mathrm{CH}_{3} \mathrm{OH}$, that are consistent with results from other star-forming regions, such as $\rho$ Oph and Perseus (Jørgensen et al. 2002, 2004, 2005). The derived fractional abundances vary by less than a factor of two among the three sources for the majority of molecular species. In the present analysis, it is not possible to correlate the abundances with the identified protostellar class. It is interesting to

\footnotetext{
${ }^{2}$ http://www. strw.leidenuniv.nl/ moldata
} 
Table 2. Model results.

\begin{tabular}{|c|c|c|c|c|}
\hline Molecule & $\frac{\text { IRS7A }}{f(\mathrm{X}) / f\left(\mathrm{H}_{2}\right)}$ & $\frac{\text { IRS7B }}{f(\mathrm{X}) / f\left(\mathrm{H}_{2}\right)}$ & $\begin{array}{c}\text { IRAS32 } \\
f(\mathrm{X}) / f\left(\mathrm{H}_{2}\right)\end{array}$ & $\frac{\text { Standard }^{a}}{f(\mathrm{X}) / f\left(\mathrm{H}_{2}\right)}$ \\
\hline $\mathrm{C}^{18} \mathrm{O}$ & $6.0 \times 10^{-8}$ & $5.0 \times 10^{-8}$ & $1.0 \times 10^{-7}$ & $4.1 \times 10^{-8}$ \\
\hline $\mathrm{C}^{17} \mathrm{O}$ & $2.0 \times 10^{-8}$ & $1.5 \times 10^{-8}$ & $2.5 \times 10^{-8}$ & $1.4 \times 10^{-8}$ \\
\hline $\mathrm{C}^{34} \mathrm{~S}$ & $5.0 \times 10^{-11}$ : & $5.0 \times 10^{-11}$ : & $\ldots$ & $5.1 \times 10^{-11}$ \\
\hline $\mathrm{CS}$ & $8.0 \times 10^{-10}$ & $6.0 \times 10^{-10}$ & $1.0 \times 10^{-9}$ : & $2.5 \times 10^{-9}$ \\
\hline $\mathrm{HCN}$ & $\ldots$ & $\ldots$ & & $2.2 \times 10^{-9}$ \\
\hline HNC & $1.0 \times 10^{-10}$ & $1.0 \times 10^{-10}$ & $1.2 \times 10^{-10}$ & $2.5 \times 10^{-10}$ \\
\hline $\mathrm{C}_{2} \mathrm{H}$ & $5.0 \times 10^{-10}$ & $4.0 \times 10^{-10}$ & $9.0 \times 10^{-10}$ & $\ldots$ \\
\hline $\mathrm{p}-\mathrm{H}_{2} \mathrm{CO}$ & $2.0 \times 10^{-10}$ & $1.0 \times 10^{-10}$ & $4.0 \times 10^{-11}$ & $6.9 \times 10^{-10}$ \\
\hline $\mathrm{A}-\mathrm{CH}_{3} \mathrm{OH}$ & $6.0 \times 10^{-10}$ & $7.0 \times 10^{-10}$ & $<7.0 \times 10^{-11}$ & $1.8 \times 10^{-9}$ \\
\hline $\mathrm{E}-\mathrm{CH}_{3} \mathrm{OH}$ & $7.0 \times 10^{-10}$ & $6.0 \times 10^{-10}$ & $<1.3 \times 10^{-10}$ & $1.3 \times 10^{-9}$ \\
\hline
\end{tabular}

${ }^{a}$ Median values from Jørgensen et al. (2002), Jørgensen et al. (2004), and Jørgensen et al. (2005). Note that $\mathrm{CH}_{3} \mathrm{OH}$ was only detected in known outflow sources. A colon (:) marks an adopted value used to set the density scale.

note that the abundances of both $\mathrm{H}_{2} \mathrm{CO}$ and $\mathrm{CH}_{3} \mathrm{OH}$ vary by about an order of magnitude among the three sample sources, possibly indicating a difference in central heating source or impact of outflows. We find the abundances of these molecules to be particularly low in IRAS32.

IRS7A and IRS7B form a protobinary system with a separation of about $18^{\prime \prime}(3000 \mathrm{AU})$. As such, RCrA-IRS7 is also a cousin of the well-studied IRAS 16293-2422 and NGC-IRAS4 binaries in terms of the total luminosity. The fact that Jørgensen et al. (2005) only detected $\mathrm{CH}_{3} \mathrm{OH}$ emission from known outflow sources in their large survey of protostellar cores, together with the low kinetic temperature $(\approx 20 \mathrm{~K})$ we derive from our observed $\mathrm{CH}_{3} \mathrm{OH}$ emission in contrast to what we get for $\mathrm{H}_{2} \mathrm{CO}$ $(\approx 40-60 \mathrm{~K})$, suggests the impact of outflows in regulating the chemistry around IRS7. These temperatures are similar to those obtained for NGC-IRAS4 (Maret et al. 2004, 2005), but lower than those for IRAS 16293-2422 (van Dishoeck et al. 1995). However, in contrast to the NGC-IRAS4A binary in particular, IRS7 does not show $\mathrm{CH}_{3} \mathrm{OH}$ lines that are broader than other observed molecular lines. Nonetheless, a large scale outflow thought to be associated with IRS7 has been observed in several other molecular species (Levreault 1988; Anderson et al. 1997b).

To make further progress, in particular to solidify the claims regarding the chemistry in the direct neighbourhood of the observed protostars, more detailed knowledge of the underlying physical structure is required. Such work is in progress and involves mid-IR observations from large ground-based telescopes and the Spitzer space telescope, and interferometric $\mathrm{mm} / \mathrm{sub}-\mathrm{mm}$ observations from ATCA and the SMA.

Acknowledgements. We would like to thank the anonymous referee who helped us improve the presentation of this work. The authors are grateful to the staff at the APEX telescope. F.L.S. acknowledges financial support from the Swedish Research Council. The research of J.K.J. was supported by the NASA Origins Grant NAG5-13050.

\section{References}

Anderson, I. M., Harju, J., \& Haikala, L. K. 1997a, A\&A, 326, 366

Anderson, I. M., Harju, J., Knee, L. B. G., \& Haikala, L. K. 1997b, A\&A, 321, 575

Brown, A. 1987, ApJ, 322, L31

Cazaux, S., Tielens, A. G. G. M., Ceccarelli, C., et al. 2003, ApJ, 593, L51

Ceccarelli, C., Loinard, L., Castets, A., Tielens, A. G. G. M., \& Caux, E. 2000, A\&A, 357, L9

Chini, R., Kämpgen, K., Reipurth, B., et al. 2003, A\&A, 409, 235

Jansen, D. J., van Dishoeck, E. F., \& Black, J. H. 1994, A\&A, 282, 605

Jayawardhana, R., Hartmann, L., \& Calvet, N. 2001, ApJ, 548, 310

Jørgensen, J. K., Schöier, F. L., \& van Dishoeck, E. F. 2002, A\&A, 389, 908 Jørgensen, J. K., Schöier, F. L., \& van Dishoeck, E. F. 2004, A\&A, 416, 603 Jørgensen, J. K., Schöier, F. L., \& van Dishoeck, E. F. 2005, A\&A, 437, 501

Knude, J. \& Høg, E. 1998, A\&A, 338, 897

Levreault, R. M. 1988, ApJS, 67, 283

Maret, S., Ceccarelli, C., Caux, E., et al. 2004, A\&A, 416, 577

Maret, S., Ceccarelli, C., Tielens, A. G. G. M., et al. 2005, A\&A, 442, 527

Nutter, D. J., Ward-Thompson, D., \& André, P. 2005, MNRAS, 357, 975

Schöier, F. L., Jørgensen, J. K., van Dishoeck, E. F., \& Blake, G. A. 2002, A\&A, 390, 1001

Schöier, F. L., Jørgensen, J. K., van Dishoeck, E. F., \& Blake, G. A. 2004, A\&A, 418,185

Schöier, F. L., van der Tak, F. F. S., van Dishoeck, E. F., \& Black, J. H. 2005, A\&A, 432, 369

van Dishoeck, E. F., Blake, G. A., Jansen, D. J., \& Groesbeck, T. D. 1995, ApJ, 447, 760 